\title{
A THEOREM OF DICKSON ON IRREDUCIBLE POLYNOMIALS
}

\section{CARLITZ}

1. Introduction. Let $p>2, n \geqq 1$. In 1911 Dickson [3] studied the distribution of irreducible cubics

$$
Q(x)=x^{3}-x^{2}+a x+b^{2} \quad\left(a, b \in G F\left(p^{n}\right)\right) .
$$

Using elementary methods he determined the number of such irreducibles. (The results are reproduced in $\$ 5$ below.)

In the present paper we consider the more general problem of irreducibles

$$
Q(x)=x^{m}+c_{1} x^{m-1}+\cdots+c_{m} \quad\left(c_{i} \in G F\left(p^{n}\right)\right)
$$

with preassigned $c_{1}, c_{m}$; for brevity we shall call $c_{1}$ and $c_{m}$ the first and last coefficients, respectively, of $Q(x)$. We first derive an asymptotic formula for the number of such irreducibles, namely,

$$
\frac{1}{m} p^{n(m-2)}+O\left(p^{n m / 2}\right) \quad(m \rightarrow \infty) .
$$

It is of interest to note that for the $L$-functions arising in this connection the Riemann hypothesis is very easily proved (compare [2]).

In the second place we consider irreducibles $Q(x)$ with assigned first coefficient and last coefficient equal to a square (or a nonsquare) of the field, thus directly generalizing Dickson's problem. We now obtain exact results (see (5.6) below). Finally we determine the number of irreducibles with assigned first coefficient.

2. For $a \in G F\left(p^{n}\right)$ we put

$$
E(a)=e^{2 \pi i t(a) / p}, \quad t(a)=a+a^{p}+\cdots+a^{p^{n-1}} .
$$

Then if $M=x^{m}+a_{1} x^{m-1}+\cdots+a_{m}\left(a_{i} \in G F\left(p^{n}\right)\right)$, and $b$ is an arbitrary number of $G F\left(p^{n}\right)$, we define

$$
\lambda(M)=\lambda_{b}(M)=E\left(b a_{1}\right) \quad(\operatorname{deg} M \geqq 1) ;
$$

also $\lambda(1)=1$. There are thus $p^{n}$ functions $\lambda$; in particular $\lambda_{0}(M) \equiv 1$. We note that

$$
\sum_{\lambda} \lambda(M)= \begin{cases}p^{n} & \left(a_{1}=0\right) \\ 0 & \left(a_{1} \neq 0\right)\end{cases}
$$

Presented to the Society, April 26, 1952; received by the editors January 15, 1952. 
where the sum in the left member is extended over all $\lambda$.

In the next place let $\boldsymbol{\gamma}$ be a primitive root of the field. Then if $a=\gamma^{r}$, we put

$$
X(a)=e^{2 \pi i r /(p m-1)}, \quad X(0)=0 .
$$

If now $c$ is an integer, $0 \leqq c<p^{n}-1$, we define

$$
\chi(M)=\chi_{c}(M)=X\left(a_{m}^{c}\right) .
$$

There are $p^{n}-1$ functions $\chi$; in particular, $\chi_{0}(M) \equiv 1$ for $x \nmid M$. We note that

$$
\sum_{\chi} \chi(M)= \begin{cases}p^{n}-1 & \left(a_{m}=1\right), \\ 0 & \left(a_{m} \neq 1\right) .\end{cases}
$$

It is clear from the definitions that

$$
\lambda(A B)=\lambda(A) \lambda(B), \quad \chi(A B)=\chi(A) \chi(B)
$$

and for $m \geqq 1$

$$
\begin{array}{ll}
\sum_{\operatorname{deg} M=m} \lambda(M)=0 & \left(\lambda \neq \lambda_{0}\right), \\
\sum_{\operatorname{deg} M=m} \chi(M)=0 & \left(\chi \neq \chi_{0}\right) .
\end{array}
$$

3. We next define the function

$$
L(s, \lambda, \chi)=\sum_{M} \lambda(M) \chi(M)|M|^{-s} \quad\left(|M|=p^{n \operatorname{des} M}\right),
$$

the sum extending over all primary $M \in G F\left[p^{n}, x\right]$. If we put

$$
\tau_{m}=\tau_{m}(\lambda, \chi)=\sum_{\operatorname{deg} M=m} \lambda(M) \chi(M),
$$

it is clear that (3.1) implies

$$
L(s, \lambda, \chi)=\sum_{m=0}^{\infty} \tau_{m}(\lambda, \chi) p^{-n m s} .
$$

Now in the first place

$$
L\left(s, \lambda_{0}, \chi_{0}\right)=\sum_{x \chi_{M}}|M|^{-s}=\left(1-p^{-n s}\right)\left(1-p^{n(1-s)}\right)^{-1} .
$$

Secondly for $\chi \neq \chi_{0}$, by the second of (2.7),

$$
L\left(s, \lambda_{0}, \chi\right)=\sum_{M} \chi(M)|M|^{-\bullet}=1 ;
$$

similarly for $\lambda \neq \lambda_{0}$ by the first of (2.7) 


$$
L\left(s, \lambda, \chi_{0}\right)=\sum_{M} \lambda(M)|M|^{-\bullet}=1-p^{-n s} .
$$

It remains to consider $L(s, \lambda, \chi)$, where $\lambda \neq \lambda_{0}, \chi \neq \chi_{0}$. We remark first that by (2.2) and (2.4)

$$
\tau_{1}=\tau_{1}(\lambda, \chi)=\sum_{a \in G F\left(p^{n}\right)} E(b a) X\left(a^{c}\right) ;
$$

then we have the easily proved formula [2]

$$
\left|\tau_{1}\right|=p^{n / 2} \quad\left(\lambda \neq \lambda_{0}, \chi \neq \chi_{0}\right) .
$$

As for $n>1$, it is evident from (3.2) that

$$
\tau_{m}(\lambda, \chi)=p^{n(m-2)} \sum_{a_{1}, a_{m}} E\left(b a_{1}\right) X\left(a_{m}^{c}\right)
$$

thus

$$
\tau_{m}(\lambda, \chi)=0 \quad\left(\lambda \neq \lambda_{0}, \chi \neq \chi_{0}, m>1\right) .
$$

Hence by (3.3) and (3.8), we have

$$
L(s, \lambda, \chi)=1+\tau_{1}(\lambda, \chi) p^{-n \varepsilon} \quad\left(\lambda \neq \lambda_{0}, \chi \neq \chi_{0}\right) .
$$

4. Returning to (3.1) it is clear that

$$
L(s, \lambda, \chi)=\prod_{P}\left(1-\lambda(P) \chi(P)|P|^{-s}\right)^{-1},
$$

the product extending over all (primary) irreducibles $P \in G F\left[p^{n}, x\right]$. Taking logarithms, we get

$$
\log L(s, \lambda, \chi)=\sum_{P} \sum_{r=1}^{\infty} \frac{1}{r} \lambda\left(P^{r}\right) \chi\left(P^{r}\right)|P|^{-r s} .
$$

Now let $a, l$ be fixed numbers of $G F\left(p^{n}\right), l \neq 0$. Then by (2.3) and (2.5), (4.2) implies

$$
\text { (4.3) } \sum_{\lambda, x} E(-b a) X\left(l^{-c}\right) \log L(s, \lambda, \chi)=p^{n}\left(p^{n}-1\right) \sum_{P, r} \frac{1}{r}|P|^{-r s} \text {, }
$$

where in the right member of (4.3) the summation is restricted to $P$ and $r$ such that $P^{r}$ has first coefficient $a$ and last coefficient $l$. Let $\pi(t, r)=\pi(t, r ; a, l)$ denote the number of such $P$ of degree $t / r$.

As for the left member of (4.3), we have first from (3.4) the contribution

$$
\log L\left(s, \lambda_{0}, \chi_{0}\right)=\sum_{r=1}^{\infty} \frac{1}{r}\left(p^{n r}-1\right) p^{-n r s}
$$


In view of (3.5), $\log L\left(s, \lambda_{0}, \chi\right)=0$, but by (3.6)

$$
\log L\left(s, \lambda, \chi_{0}\right)=-\sum_{r=1}^{\infty} \frac{1}{r} p^{-n r s} .
$$

Also (3.9) yields

$$
\log L(s, \lambda, \chi)=\sum_{r=1}^{\infty} \frac{(-1)^{r-1}}{r} \tau_{1}^{r} p^{-n r s} .
$$

Thus in all we have for the left member of (4.3)

$$
\sum_{r=1}^{\infty} \frac{1}{r} p^{-n r s} W_{r}
$$

where

$$
W_{r}=p^{n r}-\sum_{b} E(-b a)+(-1)^{r-1} \sum_{\lambda \neq \lambda_{0}, \chi_{\neq \neq} \neq \chi_{0}} E(-b a) X\left(l^{-c}\right) \tau_{1}^{r}(\lambda, \chi) .
$$

Hence it is clear that

$$
p^{n}\left(p^{n}-1\right) \sum_{r \mid t} \frac{1}{r} \pi(t, r ; a, l)=\frac{1}{t} W_{t} .
$$

Now since on the one hand

$$
\sum_{r \mid t, r>1} \frac{1}{r} \pi(t, r ; a, l)=O\left(p^{n t / 2}\right),
$$

and on the other hand by (3.7)

$$
\sum_{\lambda \neq \lambda_{0}, x \neq x_{0}} E(-b a) X\left(l^{-c}\right) \tau_{1}^{t}(\lambda, \chi)=O\left(p^{n t / 2}\right)
$$

it is evident that (4.4) implies

$$
\pi(t, 1 ; a, l)=\frac{1}{t} p^{n(t-2)}+O\left(p^{n t / 2}\right) \quad(t \rightarrow \infty) .
$$

This proves:

THEOREM 1. The number of primary irreducibles of degree $t$ with assigned first and last coefficients $a, l, l \neq 0$, satisfies (4.5).

5. We now assume $p>2$ and consider the special case of irreducible polynomials with preassigned first coefficient and with last coefficient required to be a square (or a nonsquare) of the field. It is convenient to define a function $\psi(a), a \in G F\left(p^{n}\right),=0,+1,-1$ according as $a=0$, 
square, nonsquare. Then corresponding to $\psi$ will be a single function $\chi_{1}(M)$. The preceding discussion applies except that in place of the $\chi$ 's we now use only $\chi_{0}, \chi_{1}$. We first replace (3.7) by an exact formula. It is clear that

$$
\tau_{1}\left(\lambda, \chi_{1}\right)=\sum_{a} E(b a) \psi(a)=G(b),
$$

where $G(b)$ denotes a Gauss sum $[1, \S 3]$. We recall the following formulas

$$
G(b)=\psi(b) G(1), \quad G^{2}(1)=\psi(-1) p^{n} ;
$$

since $G(b)=0$, the first of $(5.2)$ is valid for all $b$.

In the next place it is clear that corresponding to the right member of (4.4), we get

$$
\frac{1}{t}\left\{p^{n t}-\sum_{b} E(-b a)+(-1)^{t-1} \sum_{b \neq 0} E(-b a) \psi(l) G^{t}(b)\right\} \text {. }
$$

We now use (5.2) and consider separately two cases.

(i) $t$ even. In this case the second sum in (5.3) becomes

$$
-p^{n t / 2} \psi\left((-1)^{t / 2} l\right) \sum_{b \neq 0} E(-b a) \text {; }
$$

the sum in (5.4) $=p^{n}-1$ or -1 according as $a=0$ or $\neq 0$.

(ii) $t$ odd. In this case the sum in (5.3) yields

$$
\begin{aligned}
\psi(l) G^{t}(1) \sum_{b} E(-b a) \psi(b) \\
\quad=\psi(a) G^{t}(1) G(-a)=\psi\left((-1)^{(t-1) / 2} a l\right) p^{n(t+1) / 2},
\end{aligned}
$$

which holds for all $a$ (including $a=0$ ).

Now for $r \mid t$, let $\pi(t, r ; a, 1)$ denote the number of irreducibles $P$ of degree $t / r$ such that the first coefficient of $P^{r}$ is $a$, while the last is a square; $\pi(t, r ; a,-1)$ is the corresponding number with the last coefficient a nonsquare. Then as in $\$ 4$, we get, making use of (5.3), (5.4), (5.5),

\section{THEOREM 2.}

$$
\sum_{r \mid t} \frac{1}{r} \pi(t, r ; a, \eta)=\frac{1}{2 t}\left(p^{n(t-1)}-\epsilon+S\right),
$$

where $S$ is determined by

$$
S=\left\{\begin{array}{lc}
-\left(\epsilon p^{n}-1\right) p^{n(t / 2-1)} \psi\left((-1)^{t / 2} l\right) & (t \text { even }), \\
p^{n(t-1) / 2} \psi\left((-1)^{(t-1) / 2} a l\right) & (t \text { odd }),
\end{array}\right.
$$


$\epsilon=1$ for $a=0, \epsilon=0$ for $a \neq 0$, and $\eta=\psi(l)$.

Suppose now $t$ prime $\geqq 3$. Then for $t=p$

$$
\pi(t, t ; a, \eta)= \begin{cases}0 & (a \neq 0), \\ \left(p^{n}-1\right) / 2 & (a=0),\end{cases}
$$

while for $t \neq p$

$$
\pi(t, t ; a, \eta)= \begin{cases}1 & (\psi(t a l)=1) \\ 0 & \text { (otherwise) }\end{cases}
$$

In conjunction with (5.7), this gives for $a=0$

$$
\pi(t, 1 ; 0, \eta)= \begin{cases}\frac{1}{2 t}\left(p^{n(t-1)}-1\right) & (t \neq p), \\ \frac{1}{2 p}\left(p^{n(t-1)}-p^{n}\right) & (t=p),\end{cases}
$$

while for $a \neq 0$, we have

$$
\pi(t, 1 ; a, \eta)=\left\{\begin{array}{lr}
\frac{1}{2 t}\left(p^{n(t-1)}+S-2\right) & (t \neq p, \psi(t a l)=1) \\
\frac{1}{2 t}\left(p^{n(t-1)}+S\right) & \text { (otherwise) }
\end{array}\right.
$$

In Dickson's theorem, $t=3, a=1, \eta=+1$. Using the quadratic reciprocity theorem we can verify that (5.9) implies that the number of irreducible cubics satisfying these conditions is equal to

$$
\begin{array}{lr}
\left(p^{n}-1\right)\left(p^{n}+2\right) / 6 & \left(p^{n} \equiv 1(\bmod 12)\right), \\
\left(p^{n}+1\right)\left(p^{n}-2\right) / 6 & \left(p^{n} \equiv-1(\bmod 12)\right), \\
p^{n}\left(p^{n}+1\right) / 6 & \left(p^{n} \equiv 5(\bmod 12)\right), \\
p^{n}\left(p^{n}-1\right) / 6 & \left(p^{n} \equiv-5(\bmod 12)\right),
\end{array}
$$

while for $p=3$ we get

$$
\begin{array}{lr}
p^{n}\left(p^{n}+1\right) / 6 & (n \text { even }), \\
p^{n}\left(p^{n}-1\right) / 6 & (n \text { odd }) .
\end{array}
$$

These results check with Dickson's, thus affording a partial check for the more general formulas derived above.

6. It may be worth while mentioning briefly the formula for the number of irreducibles of degree $t$ with given first coefficient $a$. If 
$p \nmid t$, then it is clear (by considering $P(x+b)$ ) that the number of irreducibles is independent of $a$ and is therefore $f(t) / p^{n}$, where $f(t)=f\left(t, p^{n}\right)$ is the total number of (primary) irreducibles of degree $t$. For $p \mid t$, the transformation $c^{t} P\left(c^{-1} x\right)$ indicates that the number of irreducibles with first coefficient $a$ is independent of $a$ provided $a \neq 0$, but this gives no information for the case $a=0$. Accordingly we make use of the $\lambda$ 's defined above and set up

$$
L(s, \lambda)=\sum_{M} \lambda(M)|M|^{-\bullet}= \begin{cases}\left(1-p^{n(1-s)}\right)^{-1} & \left(\lambda=\lambda_{0}\right), \\ 1 & \left(\lambda \neq \lambda_{0}\right) .\end{cases}
$$

For $r \mid t$, let $\pi(t, r ; a)$ denote the number of irreducibles $P$ of degree $t / r$ such that the first coefficient of $P^{r}$ is $a$. Then exactly as in $\$ \$ 4,5$ we find that

$$
\sum_{r \mid t} \frac{1}{r} \pi(r, t ; a)=\frac{1}{t} p^{n(t-1)} .
$$

If $t=p$, it is evident that $\pi(p, p ; a)=0$ for $a \neq 0$, while $\pi(p, p ; 0)$ $=p^{n}$. Hence (6.1) implies

$$
\pi(p, 1 ; a)= \begin{cases}p^{n-n-1} & (a \neq 0), \\ p^{n p-n-1}-p^{n-1} & (a=0) .\end{cases}
$$

We now determine $\pi(t, 1 ; a)$ for arbitrary $t$; we need only consider the case $p \mid t$. Now it is clear $\pi(t, r ; a)=0$ for $p \mid r, a \neq 0$, while $\pi(t, r ; 0)$ $=f(t / r)$ for $p \mid r$. On the other hand for $p \nmid r$, we have $\pi(t, r ; a)$ $=\pi(t / r, 1 ; a / r)$ for all $a$. Now let $t=p^{k} m, p \nmid m$, and consider (6.1) with $a=1$. Then it is evident that we need only take such $r$ for which $p \nmid r$; thus (6.1) becomes

$$
\sum_{r \mid m} \frac{1}{r} \pi(t, r ; 1)=\frac{1}{t} p^{n(t-1)},
$$

which reduces to

$$
\sum_{r \mid m} \frac{1}{r} \pi(t / r, 1 ; 1)=\frac{1}{t} p^{n(t-1)},
$$

or what is the same thing

$$
p^{n+k} \sum_{d \mid m} d \pi\left(p^{k} d, 1 ; 1\right)=p^{n p^{k m}} .
$$

Comparing (6.3) with the familiar equation

$$
\sum_{d \mid m} d f\left(d, p^{n}\right)=p^{n m}
$$


which has the unique solution $f\left(d, p^{n}\right)$, it follows that

$$
p^{n+k} \pi\left(p^{k} m, 1 ; 1\right)=f\left(m, p^{n p^{k}}\right) .
$$

Since $\left(p^{n}-1\right) \pi(m, 1 ; 1)+\pi(m, 1 ; 0)=f(m)$, we have also

(6.5) $\quad p^{n+k} \pi\left(p^{k} m, 1 ; 0\right)=p^{n+k} f\left(p^{k} m, p^{n}\right)-\left(p^{n}-1\right) f\left(m, p^{n p^{k}}\right)$.

It is easily verified that for $k=1, m=1,(6.4)$ and (6.5) reduce to (6.2).

THEOREM 3. The number of primary irreducible polynomials of degree $p^{k} m$ and assigned first coefficient is determined by (6.4) and (6.5).

\section{REFERENCES}

1. L. Carlitz, The singular series for sums of squares of polynomials, Duke Math. J. vol. 14 (1947) pp. 1105-1120.

2. H. Davenport and H. Hasse, Die Nullstellen der Kongruenzzetafunktionen in gewissen zyklischen Fällen, J. Reine Angew. Math. vol. 172 (1935) pp. 151-182.

3. L. E. Dickson, $A n$ invariantive investigation of irreducible binary modular form, Trans. Amer. Math. Soc. vol. 12 (1911) pp. 1-18.

DUKe UNIVERSITY 